\title{
Green's function for magnetically incoherent interacting electrons in one dimension
}

\author{
Gregory A. Fiete ${ }^{1,2,3}$ and Leon Balents ${ }^{3}$ \\ ${ }^{1}$ Lyman Laboratory of Physics, Harvard University, Cambridge, MA 02138, USA \\ ${ }^{2}$ Kavli Institute for Theoretical Physics, University of California, Santa Barbara, CA 93106, USA \\ ${ }^{3}$ Physics Department, University of California, Santa Barbara, CA 93106, USA
}

(Dated: December 2, 2018)

\begin{abstract}
Using a path integral approach and bosonization, we calculate the low energy asymptotics of the one particle Green's function for a "magnetically incoherent" one dimensional strongly interacting electron gas at temperatures much greater than the typical exchange energy but much lower than the Fermi energy. The Green's function exhibits features reminiscent of spin-charge separation, with exponential spatial decay and scaling behavior with interaction dependent anomalous exponents inconsistent with any unitary conformal field theory. We compute the tunneling density of states at low energies and find that it is a power law in energy with exponent $1 /(4 g)-1$, where $g$ is the Luttinger interaction parameter in the charge sector. The underlying physics is made transparent by the simplicity of the approach. Our results generalize those of Cheianov and Zvonarev [Phys. Rev. Lett. 92, 176401 (2004)].

PACS numbers: 71.10.-w,71.10.Pm,71.27.+a,73.21.-b
\end{abstract}

Interacting one dimensional electron systems have proven to be especially rich in their physics, most notably because of the universal low energy properties that are present when the interactions are not too strong, the so-called Luttinger Liquid (LL) state 1 whose existence in nature is now established [2]. It is well known [3] that the LL state exhibits decoupled spin and charge degrees of freedom (spin-charge separation) with distinct spin and charge velocities for the respective collective modes. Characteristic of the LL state are power-law decays of various correlation functions, notably the singleparticle Green's function, which is suppressed relative to the naïve expectation of Fermi liquid theory. As one consequence, the local tunneling density of states vanishes in a power-law fashion as the chemical potential is approached. All low-energy properties of the LL state can be understood from bosonization, which relates them to correlators in a simple free boson unitary conformal field theory (CFT).

In the regime of strongly interacting, very low density electrons, different physics is to be expected when the assumptions of LL theory break down. The distinction between low and high densities is often quantified by the parameter $r_{s} \equiv\left(\bar{n} a_{B}\right)^{-1}$ where $\bar{n}$ is the average electron density and $a_{B}$ is the Bohr radius specific to the material. When $r_{s} \gg 1$ the spacing between electrons is large compared to $a_{B}$ and the potential energy dominates the kinetic energy, driving the system towards a Wigner crystal. When such strong interactions are present, it becomes difficult for electrons to exchange their position since they must effectively tunnel through one another, leading to an exponentially small $\left[4,[5]\right.$ (in $r_{s}$ ) exchange energy, $J$. It then becomes quite easy to reach the magnetically incoherent regime where the exchange energy is much less than the temperature: $J \ll T$. In this Letter we compute the low energy asymptotics of the 1-d Green's function for general interactions in the limit $r_{s} \gg 1$ when the temperature is still much less than the Fermi energy: $J \ll T \ll E_{F}$. We find that the Green's functions exhibits exponential decay in the spin sector and power law decay in the charge sector characterized by interaction dependent anomalous exponents (which do not correspond to any unitary conformal field theory). Our results generalize the results of Cheianov and Zvonarev [ [6] (CZ) and the zero field results of Berkovich [7] and are obtained in a much simpler and more physically transparent manner.

Due to breakthroughs in materials technology that allow unprecedented exploration of clean 1-d quantum wires [8] theoretical interest in such systems has also been renewed $[9]$. The regime $J \ll T \ll E_{F}$ was recently considered by Matveev [5] where he showed there is a drop in the conductance of a one channel wire from $2 e^{2} / h$ to $e^{2} / h$ when $J$ drops below $T$. In the same regime CZ 6$]$ have computed the low energy asymptotics of the one particle Green's function assuming infinitely strong zero range interactions between electrons. Their tour-de-force Betheansatz based calculation, however, does not provide clear insight into the physics. Moreover, their results are specific to the special features of the Hamiltonian they considered. Our calculation of the 1-d Green's function in the regime $J \ll T \ll E_{F}$ is completely general and simple enough to highlight the physical origins of the non-LL features.

We note that the physics of the $J \ll T \ll E_{F}$ regime is of broad and general interest as it may play some role in other systems not fully understood, such as the two dimensional metal-insulator transition and the 0.7 anomaly in quantum point contacts. As such, it is a worthwhile endeavor to elucidate the basic physics of this regime.

MODEL: We assume that the electrons are confined to one dimension and experience predominantly repulsive and spin independent interactions, i.e. ones which can be written solely in terms of the local electron den- 
sity $n(x)=\sum_{\sigma=\uparrow, \downarrow} \psi_{\sigma}^{\dagger}(x) \psi_{\sigma}(x)$. Here $\psi_{\sigma}(x)$ is the field operator for an electron at position $x$ with spin $\sigma$. In the discussion that follows, we will not require an explicit Hamiltonian, but rather make use of an effective lowenergy theory that contains renormalized parameters, $v_{c}$ and $g$, depending on the microscopic interactions in an unspecified way. Despite our interest in the magnetically incoherent regime $J \ll T$, we will see that the essential parameters describing the effect of interactions may be obtained from the charge sector action of LL theory which obtains at the lowest temperatures $T \ll J$ :

$$
S_{c}=\int d x d \tau \frac{v_{c}}{2 \pi}\left[\frac{1}{2 g}\left(\partial_{x} \theta\right)^{2}+2 g\left(\partial_{x} \phi\right)^{2}\right]+\frac{i}{\pi} \partial_{\tau} \phi \partial_{x} \theta,
$$

where $v_{c}$ is the velocity of charge excitations, $g$ is the parameter of the low energy theory measuring the strength of electron interactions and the charge fields $\theta$ and $\phi$ are the fields appearing in the low-temperature bosonized version of the electron operator. The charge fields are defined via $\theta_{\uparrow}+\theta_{\downarrow}=\theta$ and $\left(\phi_{\uparrow}+\phi_{\downarrow}\right) / 2=\phi$. The $\theta$ field is related to the particle density fluctuations through the familar relationship $n(x)=\frac{1}{\pi} \partial_{x} \theta(x)$ and $e^{\pi \phi(x)}$ annihilates/creates a particle at $\mathrm{x}$. We choose to do all our calculations in imaginary time, $\tau=i t$. The Green's functions of interest (retarded, advanced, etc.) can then be computed by the appropriate analytical continuation to real time.

RESULTS: We compute the single particle Green's function

$$
\mathcal{G}(x, \tau)=\left\langle\psi_{\uparrow}(x, \tau) \psi_{\uparrow}^{\dagger}(0,0)\right\rangle,
$$

in the limit $J \ll T \ll E_{F}$ by first averaging over spin configurations and then computing the low energy charge dynamics using (11). Due to the spin rotational invariance of the electron interactions, the Green's function for spin down electrons coincides with (2). We find (for $x>0, \tau>0 \rightarrow \infty)$,

$\mathcal{G}(x, \tau)=\frac{C^{\prime} e^{-\tilde{k}_{F} x \frac{\ln 2}{\pi}}}{\left(x^{2}+v_{c}^{2} \tau^{2}\right)^{\Delta_{g}}}\left(\frac{e^{i\left(\tilde{k}_{F} x-\varphi_{g}^{+}\right)}}{v_{c} \tau-i x}+\frac{e^{-i\left(\tilde{k}_{F} x-\varphi_{g}^{-}\right)}}{v_{c} \tau+i x}\right)$

where $C^{\prime}$ is an undetermined constant $(\mathrm{CZ}$ determine it for the special case of infinite strength zero range interactions in Ref. [6]). Here we follow CZ and define a spinless Fermi wavevector $\tilde{k}_{F} \equiv \pi \bar{n}$, where $\bar{n}$ is the average density of electrons. The phases $\varphi_{g}^{ \pm}$are given by

$$
\varphi_{g}^{ \pm}(x, \tau)=\frac{\ln 2}{\pi}\left(g \ln \left(x^{2}+v_{c}^{2} \tau^{2}\right) \pm \frac{1}{2} \ln \left(\frac{v_{c} \tau-i x}{v_{c} \tau+i x}\right)\right) .
$$

The power law decay of (3) is characterized by the anomalous exponent

$$
\Delta_{g}=\frac{1}{8 g}+\frac{g}{2}\left(1-\left(\frac{\ln 2}{\pi}\right)^{2}\right)-\frac{1}{2} .
$$

It is clear that the Green's function (3) does not fit into the usual LL paradigm of correlation functions with power law decay because of the exponential decay with distance. We will show that this exponential decay is simply a result of spin averaging when $J \ll T$. The exponents characterizing the power law decay of the charge sector are anomalous because for certain values of $g$, $g=1 / 2$ for example, $\Delta_{g=\frac{1}{2}}=-\frac{1}{4}\left(\frac{\ln 2}{\pi}\right)^{2}<0$, which, if interpreted as arising from $\mathrm{CFT}$, implies non-unitarity in the charge sector $[\underline{6}$. We will show the anomalous power law decay of (3) comes from density fluctuations in the charge sector after averaging over the spin degrees of freedom.

We also compute the $x=0$ Green's function

$$
\mathcal{G}(0, \tau) \sim \frac{1}{\sqrt{\tau^{\frac{1}{2 g}} \ln (\tau)}},
$$

from which the low frequency spectral function can be computed

$$
A(\omega) \propto \omega^{\frac{1}{4 g}-1} /|\sqrt{\ln (w)}| .
$$

All our results recover the results of $\mathrm{CZ}$ in the special case they considered of infinite repulsive local $(\delta$ function) interactions, which is just the infinite $U$ limit of the Hubbard model. The CZ limit corresponds 10 to $g=1 / 2$ from which all their results can be recovered by plugging this value of $g$ into Eqs. (4)-(17).(CZ ignore the $\sqrt{\ln (w)}$ factor in (7); our results agree exactly at $\mathrm{g}=1 / 2$.)

DERIVATION OF RESULTS: We study the singleparticle Green's function for a general (finite range and strength of interactions), strongly interacting 1-d electron system at finite temperature:

$$
\mathcal{G}(x, \tau)=\frac{1}{Z} \operatorname{Tr}\left[e^{-\beta H} \psi_{\uparrow}(x, \tau) \psi_{\uparrow}^{\dagger}(0,0)\right], \tau>0 .
$$

Here $Z \equiv \operatorname{Tr}\left[e^{-\beta H}\right]$ is the partition function and $\beta$ is the inverse temperature. Our results are based on the first quantized path integral representation of $\mathcal{G}(x, \tau)$ and $Z$ in imaginary time, $\tau=i t$. As is well-known, $Z$ is obtained as an integral over world lines (paths) of up and down spin electrons, with periodic boundary conditions in imaginary time allowing for permutations of identical particles, i.e. the final positions of electrons of a given spin polarization must be a permutation of their initial positions (up and down electrons may not be permuted as they are not identical). Each such configuration is weighted by $(-1)^{P_{\uparrow}+P_{\downarrow}} e^{-S_{e}}$, where $S_{e}$ is the Euclidean action describing the "deformation" of the world lines and $(-1)^{P_{\sigma}}$ is the sign of the permutation of spin $\sigma$ electrons. The numerator of Eq. (8) is a similar integral over paths, with a spin-up electron world line inserted at $(0,0)$ and another removed at $(x, \tau)$. To compute the sign of the permutation, one should treat the path terminating at $(x, \tau)$ as continuing from $(0,0)$. 
A crucial element of our analysis is a non-crossing condition. Due to the Pauli principle, world lines of the same spin electrons can be treated as non-crossing irrespective of interactions. Moreover, the condition $J \ll T$ precisely corresponds to the absence of crossings (exchanges) of opposite spin world lines $(1 / J \gg \beta$ gives the typical distance in imaginary time between exchange events). Physically, this is due to the large Coulomb repulsion of electrons. Thus the topology of the paths is identical to that of spinless fermions or infinitely repulsive spinless bosons. Some world line trajectories are shown in Fig. [1]

We choose to compute the trace in Eq. (8) by first summing over all possible spin assignments for each set of world lines. This gives an effective weight for the remaining sum over the world line configurations. This weight includes two contributions: (1) the Euclidean action factor $e^{-S_{e}}$, and (2) a magnetic/statistical factor obtained from the spin sum and permutation signs, which is discussed below. Given the non-crossing topology and spin-independent interactions, the Euclidean action is expected to be well-approximated (for $T \ll E_{F}$ ) by the low-energy effective form appropriate to spinless (in general interacting) fermions/bosons, which justifies Eq. (11). Moreover, for $J \ll E_{F}$, we expect this "charge sector" action to be identical to the ultimate low-energy charge action of LL theory valid for $T \ll J$.

Next consider the spin sum. The limit $J \ll T$ implies that for a fixed set of world lines all spin configurations are computed with equal weight. In order for a particle created at spacetime coordinate $(0,0)$ to be annihilated at $(x, \tau)$ a large distance away, the world line must "wrap around" the imaginary time inverval 0 to $\beta$ a large number of times. From Fig. 1 it is clear that to stay in the low energy sector, all intermediate world lines must have the same spin. Any configuration of world lines without all the intermediate lines having the same spin would violate the non-crossing condition. When the spin average is taken in the trace in (8), only the term with all intermediate worldlines having spin up will contribute to the low energy Green's function. For $N$ intermediate world lines, the probability that all $N+1$ spins are the same is $2^{-N}$. After an electron added at $(0,0)$ reaches $(x, \tau)$, it has been permuted through $N$ electrons of the same spin and therefore the Green's function picks up a factor $(-1)^{N}$.

After spin averaging for $J \ll T$, the Green's function therefore becomes

$$
\mathcal{G}(x, \tau) \sim\left\langle 2^{-N(x, \tau)}(-1)^{N(x, \tau)} e^{i(\phi(x, \tau)-\phi(0,0))}\right\rangle,
$$

where now the average is taken at $T \rightarrow 0$ in the charge sector. The term $e^{i(\phi(x, \tau)-\phi(0,0))}$ creates a world line at $(0,0)$ and annihilates it at $(x, \tau)$. (Note that at this stage in the calculation the dynamics after spin averaging become effectively spinless as discussed earlier.) All the effects of statistics and spin are encapsulated in the first

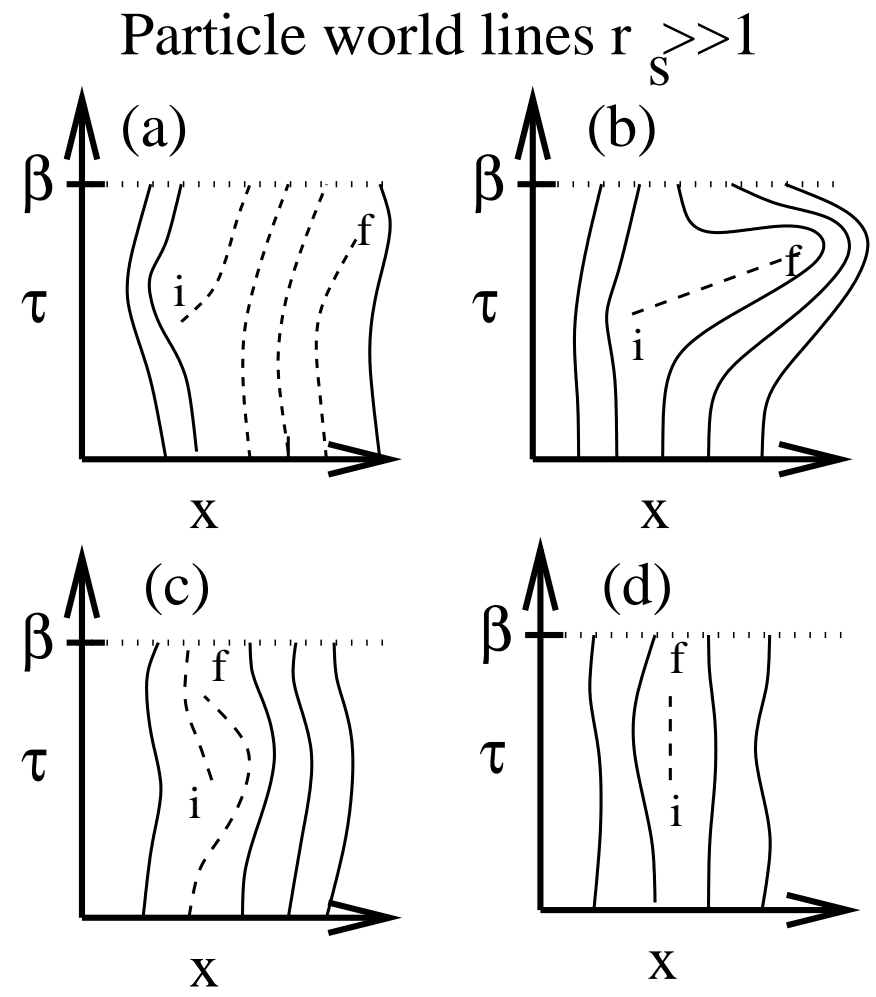

FIG. 1: World lines for a strongly interacting 1-d electron gas at $J<<T$. Particle trajectories in space and imaginary time are shown as curved lines. The dashed lines represent the world line paths for creating a particle and removing it for large $x=x_{f}-x_{i}, \tau=\tau_{f}-\tau_{i}$, ((a) and (b)), and for $x=0, \tau((\mathrm{c})$ and $(\mathrm{d}))$. The solid lines represent trajectories of other particles. Due to the large action cost associated with the trajectories in (b), at low energies a process like that shown in (a) where world lines "wrap around" from $\tau=\beta$ to $\tau=0$ will dominate. Such a process, however, requires that all dashed world lines have the same spin. For $J \ll T$ this occurs with probability $2^{-N}$ as discussed in the text. Fig.(c) shows a contribution to the $k=1$ term of Eq. (13), however processes like that shown in (d) dominate and yield the Green's function (6).

two terms inside the average. The number of electrons is related to the $\theta$ field via

$$
N(x, \tau)=\bar{n} x+\frac{1}{\pi}(\theta(x, \tau)-\theta(0,0)),
$$

which expressed the number of electrons in a distance $x$ in terms of the average density and a small fluctuating piece expressed in terms of the $\theta$ fields. Using Eq. (10), and writing $(-1)^{N}=\operatorname{Re}\left[e^{i \pi N}\right]$, the simplest form correct for integer $N$ (the harmonic approximation violates this) and consistent with the requirement that $\mathcal{G}(x, \tau)$ be real and even in $x$. The Green's function can now be expressed as $\mathcal{G}=\mathcal{G}_{+}+\mathcal{G}_{-}$, with $\mathcal{G}_{-}=\left[\mathcal{G}_{+}\right]^{*}$ and

$$
\begin{aligned}
\mathcal{G}_{+}(x, \tau) & \sim e^{-\tilde{k}_{F} x \frac{\ln 2}{\pi}} e^{i \tilde{k}_{F} x}\left\langle e^{-\frac{\ln 2}{\pi}(\theta(x, \tau)-\theta(0,0))}\right. \\
& \left.\times e^{i(\theta(x, \tau)-\theta(0,0))} e^{i(\phi(x, \tau)-\phi(0,0))}\right\rangle,
\end{aligned}
$$


where the first two terms come from the exponentiation of the average density and we have used $\tilde{k}_{F}=\pi \bar{n}$. This clearly identifies the exponential decay of the first term as coming from spin averaging and the oscillatory second term as coming from Fermi statistics.

We now compute the part of the Green's function coming from fluctuations in the charge sector using the action (11). Making the definitions, $\Phi(x, \tau)=\phi(x, \tau)-\phi(0,0)$ and $\Theta(x, \tau)=\theta(x, \tau)-\theta(0,0)$, we use the Gaussian action to move the averages to the exponent,

$$
\begin{aligned}
\mathcal{G}_{+}(x, \tau) \sim & e^{-\tilde{k}_{F} x \frac{\ln 2}{\pi}} e^{i \tilde{k}_{F} x}\left\langle e^{i\left(1+i \frac{\ln 2}{\pi}\right) \Theta(x, \tau)} e^{i \Phi(x, \tau)}\right\rangle \\
= & e^{-\tilde{k}_{F} x \frac{\ln 2}{\pi}} e^{i \tilde{k}_{F} x} e^{-\frac{1}{2}\left(1+i \frac{\ln 2}{\pi}\right)^{2}\left\langle\Theta^{2}\right\rangle} \\
& \times e^{-\frac{1}{2}\left\langle\Phi^{2}\right\rangle} e^{-\left(1+i \frac{\ln 2}{\pi}\right)\langle\Phi \Theta\rangle} .
\end{aligned}
$$

Standard computations from Eq. (11) give $\left\langle\Theta^{2}\right\rangle=$ $g \ln \left(x^{2}+v_{c}^{2} \tau^{2}\right),\left\langle\Phi^{2}\right\rangle=\frac{1}{4 g} \ln \left(x^{2}+v_{c}^{2} \tau^{2}\right)$ and $\langle\Phi \Theta\rangle=$ $\frac{1}{2} \ln \left(\frac{v_{c} \tau-i x}{v_{c} \tau+i x}\right)$. Substituting these values into Eq. (12), the anomalous exponent given in Eq. (5) is obtained. An additional phase factor comes from the $-\frac{\ln 2}{\pi}\left(\left\langle\Theta^{2}\right\rangle+\right.$ $\langle\Phi \Theta\rangle)=-\frac{\ln 2}{\pi}\left(g \ln \left(x^{2}+v_{c}^{2} \tau^{2}\right)+\frac{1}{2} \ln \left(\frac{v_{c} \tau-i x}{v_{c} \tau+i x}\right)\right)$ piece in the exponent. Combining this with the complex conjugate yields Eq. (3).

The preceding calculation brings out the essential physics of the Green's function (3): The spin averaging is responsible for the exponential decay of the Green's function and imposes a constraint on the world line configurations that contribute to it. Fermi statistics is responsible for the oscillatory terms. Treating Gaussian fluctuations about this constraint in the charge sector results in the power law decay with generalized anomalous exponents depending on the interaction parameter $g$.

Having discussed the spatial asymptotics of $\mathcal{G}(x, \tau)$, for $x, \tau \rightarrow \infty$, we now turn our attention to $\mathcal{G}(0, \tau)$ which will allow us to compute the low energy tunneling density of states at a point. Unlike the situation with $x \rightarrow \infty$, computing the Green's function at $x=0$ forces us to consider the discreteness in the number of world lines that may "bend' in between $(0,0)$ and $(0, \tau)$ :

$$
\begin{aligned}
\mathcal{G}(0, \tau) & \sim \sum_{k=-\infty}^{\infty} 2^{-|k|}(-1)^{k}\left\langle\delta(N(0, \tau)-k) e^{i(\phi(0, \tau)-\phi(0,0))}\right\rangle \\
& \sim \frac{1}{\sqrt{\tau^{\frac{1}{2 g}} \ln \left(v_{c} \tau\right)}} \sum_{k=-\infty}^{\infty} 2^{-|k|}(-1)^{k} e^{-\frac{\pi^{2} k^{2}}{4 g \ln \left(v_{c} \tau\right)}}, \quad(13)
\end{aligned}
$$

where the result (6) is recovered by noting that the sum depends only weakly on $\tau$ and ranges between $2 / 3$ and 1. Fourier transforming (13) into frequency space immediately gives (7).

DISCUSSION: We have computed the low energy asymptotics of the one particle Green's function $\mathcal{G}(x, \tau)$ in the limit of $r_{s} \gg 1$ and $J \ll T \ll E_{F}$ for arbitrary interactions. We find the correlation function does not fit the usual LL form. Instead, the spin averaging present in the Green's function for $J \ll T$ results in an exponential decay of the Green's function with distance. The low energy behavior of the charge sector still shows a power law decay due to Gaussian fluctuations, but with interaction dependent anomalous exponents. The low frequency tunneling density of states (proportional to the spectral function) also shows interesting behavior depending on $g: A(\omega) \propto \omega^{1 /(4 g)-1}$, which shows a crossover from a power law divergence for $g>1 / 4$, to a pseudo-gap when $g<1 / 4$. The divergence at low energies can be understood as coming from the infinite spin degeneracy when $J \ll T$. As the interactions increase ( $g$ decreases) the suppression of the density of states in the charge sector overwhelms the spin degeneracy to recover the power law suppression familiar in LL theory. Finally, we note that Fourier transforming the Green's function (at low frequency) into momentum space will result in broad peaks of width $\sim \tilde{k}_{F}$ centered at $k \approx \pm \tilde{k}_{F}$. Note that the "Fermi momentum" $\tilde{k}_{F}$ appearing here differs by a factor of 2 from the usual Fermi momentum: $k_{F}=\pi \bar{n} / 2=\tilde{k}_{F} / 2$. Hence, as one moves from $r_{s} \approx 0$ to $r_{s} \gg 1$ and the regime $J \ll T \ll E_{F}$ is reached, one expects to see delta function-like peaks present at small $r_{s}$ to broaden to width $\sim \tilde{k}_{F}$ and the centers to shift from $\pm \pi \bar{n} / 2$ to $\pm \pi \bar{n}$ creating a broad double-lobed structure in momentum resolved tunneling when $r_{s} \gg 1$ and $J \ll T \ll E_{F}$. We hope this work will inspire new ideas in other systems where the physics discussed here may play a role.

G.A.F. thanks B.I. Halperin for initially bringing Ref [6] to his attention and for emphasizing the physical richness of the $J \ll T \ll E_{F}$ regime. G.A.F. thanks B. I. Halperin, K . Le Hur, H. Steinberg, Y. Tserkovnyak and A. Yacoby for illuminating discussions. This work was supported by DMR-9985255 (L.B.), the Packard foundation (L.B.), NSF PHY99-07949 (G.A.F.) and NSF DMR 02-33773 (G.A.F.).

[1] F. Haldane, J. Phys. C 14, 2585 (1981).

[2] Y. Tserkovnyak, et al. Phys. Rev. Lett. 89, 136805 (2002). H. Ishii, et al., Nature 426, 540 (2003). M. Bockrath, et al., Nature 397, 598 (1999). Z. Yao, et al., Nature 402, 273 (1999).

[3] J. Voit, Rep. Prog. Phys. 57, 977 (1994).

[4] W. Häusler, Z. Phys. B 99, 551 (1996).

[5] K. A. Matveev, Phys. Rev. Lett. 92, 106801 (2004). K.A. Matveev, cond-mat/0405542.

[6] V. V. Cheianov and M. B. Zvonarev, Phys. Rev. Lett. 92, 176401 (2004). V. V. Cheianov and M. B. Zvonarev, J. Phys. A: Math. Gen. 37, 2261 (2004).

[7] A. Berkovich, J. Phys. A: Math. Gen. 24, 1543 (1991).

[8] O. Auslaender, et al., Science 295, 825 (2002). R. de Picciotto, et al., Phys. Rev. Lett. 92, 036805 (2004).

[9] Y. V. Nazarov and L. I. Glazman, Phys. Rev. Lett. 91, 126804 (2003). M. Pustilnik, et al., Phys. Rev. Lett. 91, 126805 (2003).

[10] H. J. Schulz, Phys. Rev. Lett. 64, 2831 (1990). 